Jozef Bobok, KM FSv ČVUT, Thákurova 7, 16629 Praha 6, Czech Republic, email: erastus@@csearn.bitnet

Milan Kuchta, Mathematical Institute, Slovak Academy of Sciences,

Štefánikova 49, 81473 Bratislava, Slovakia, email: matekuch@@savba.sk

\title{
REGISTER SHIFTS VERSUS TRANSITIVE $F$-CYCLES FOR PIECEWISE MONOTONE MAPS
}

\begin{abstract}
This paper investigates the family of continuous piecewise monotone functions which map a closed interval of the real line into itself. For these maps Preston [1] and Blokh [2] described the asymptotic behavior of the orbit of a "typical" point. Our results show that if the map is expanding on its intervals of monotonicity the dominant role is played by transitive $f$-cycles. Contrary to this for a "typical" map in a natural closure of the space of these maps there are no transitive $f$-cycles. Instead the behavior is dominated by the register shifts. This result is illustrated by an example.
\end{abstract}

\section{Introduction}

Consider a continuous function which maps a closed interval of the real line into itself. This gives us a simple dynamical system with discrete time. The new state of our system is the image of the old one using the given function. So each starting state determines a whole orbit. We are interested in the asymptotic behavior of the orbit of a "typical" point. For us "typical" has a topological rather than measure theoretic meaning.

A function is piecewise monotone if there is a finite partition of our interval such that the function is monotone on each part. If on each of these

Key Words: Iteration, Asymptotic behavior, Piecewise monotone, Expanding maps, Register shift, Transitive $f$-cycle, Residual set

Mathematical Reviews subject classification: Primary: 26A18, 26A21, 54H20, 58F08

Received by the editors December 8, 1992 
subintervals the derivative is bigger than some number greater than one, then we show that the "typical" point is attracted by a transitive $f$-cycle. This means a periodic interval in which some orbit is dense. The orbits inside this transitive $f$-cycle can be very wild. Moreover such a function does not have any register shift.

On the other hand for a "typical" function from the slightly larger space of piecewise monotone functions with derivatives greater than or equal to one (or strictly greater than one) a "typical" point is attracted by a register shift. Thus the asymptotic behavior of such an orbit is very nice and if we do not notice small differences, then it looks like a periodic orbit. It follows that the "typical" function does not have any transitive $f$-cycle. So one phenomenon is replaced by the other.

\section{Background}

Let $I=[0,1]$ and let $C(I)$ be the space of continuous functions which map $I$ into itself. This space will be endowed with the metric $\varrho$ of uniform convergence.

A function $f \in C(I)$ is called piecewise monotone if there is an $n \geq 0$ and a set of points $0=d_{0}<d_{1}<\cdots<d_{n}<d_{n+1}=1$ such that $f$ is strictly monotone on $\left[d_{k}, d_{k+1}\right]$ for each $k=0, \ldots, n$. A point $t \in(0,1)$ is called a turning point of $f$ if $f$ is not monotone in any neighborhood of $t$. We denote the set of the turning points of $f$ by $T(f)$. Let $M_{n}$ be the set of piecewise monotone functions with the number of turning points less than or equal to $n$. Let $c \geq 0, d>0$ and

$$
\begin{aligned}
& M_{n, c}=\left\{f \in M_{n} ; \text { if } f \mid[a, b] \text { is monotone, then }\left|\frac{f(b)-f(a)}{b-a}\right|>c\right\}, \\
& \tilde{M}_{n, d}=\left\{f \in M_{n} ; \text { if } f \mid[a, b] \text { is monotone, then }\left|\frac{f(b)-f(a)}{b-a}\right| \geq d\right\} .
\end{aligned}
$$

For $f \in C(I)$ define $f^{n}$ ( $n$-th iterate of $f$ ) inductively by $f^{0}(x)=x$ and (for $n \geq 1) f^{n}(x)=f\left(f^{n-1}(x)\right.$ ). The orbit of $x \in I$ with respect to $f$ is the sequence $\operatorname{orb}(x)=\left\{f^{n}(x)\right\}_{n=0}^{\infty}$. A closed interval $J \subset I$ is called periodic interval with period $\operatorname{per}(J)=k \in \mathbb{N}$ if $f^{k}(J)=J$ and $f^{i}(J) \cap f^{j}(J)=\emptyset$ for $0 \leq i \neq j<k$. If $J$ is a point, then it is called a periodic point and $\operatorname{Per}(f)$ denotes the set of all periodic points of $f$. A point $x \in I$ is called eventually periodic if $x \notin \operatorname{Per}(f)$ and $f^{k}(x) \in \operatorname{Per}(f)$ for some $k \geq 1$.

Recall that $K=\bigcup_{k=0}^{m-1} f^{k}(J)$ (the orbit of a periodic interval $J$ with pe$\operatorname{riod} m$ ) is called an $f$-cycle with period $m$. This $f$-cycle is said to be transitive 
if there is an orbit of a point which is dense in $K$. Or equivalently $K$ is transitive if for any closed $S \subset K$ such that $f(S) \subset S$ we have either $S=K$ or $\operatorname{int}(S)=\emptyset$. Note that any transitive $f$-cycle must contain a turning point. Hence a function from $M_{n}$ can have only $n$ different transitive $f$-cycles.

Let $\left\{K_{n}\right\}_{n=1}^{\infty}$ be a decreasing sequence $\left(K_{n+1} \subset K_{n}\right)$ of $f$-cycles and $m_{n}$ be a period of $K_{n}$. It is easy to see that $m_{n}$ divides $m_{n+1}$ for each $n \geq 1$. We call the sequence $\left\{K_{n}\right\}_{n=1}^{\infty}$ splitting if $m_{n+1}>m_{n}$ for each $n \geq 1$. We say that $R \subset I$ is a register shift if there is a splitting sequence of $f$-cycles $\left\{K_{n}\right\}_{n=1}^{\infty}$ such that $R=\bigcap_{n=1}^{\infty} K_{n}$. We call $\left\{K_{n}\right\}_{n=1}^{\infty}$ a generator of $R$. Again note that any register shift must contain a turning point. Hence a function from $M_{n}$ can have only $n$ different register shifts.

Let $K$ be an $f$-cycle. We define the set of attraction of $K$ by

$$
A(K, f)=\left\{x \in I: f^{n}(x) \in \operatorname{int}(K) \text { for some } n \geq 0\right\} .
$$

If $R$ is a register shift and $\left\{K_{n}\right\}_{n=1}^{\infty}$ is its generator, then similarly

$$
A(R, f)=\bigcap_{n=1}^{\infty} A\left(K_{n}, f\right)
$$

Note that $\bigcap_{n=1}^{\infty} A\left(K_{n}, f\right)=\bigcap_{n=1}^{\infty} A\left(\tilde{K}_{n}, f\right)$ for any two generators of $R$. Hence $A(R, f)$ is well defined. Finally we define the set

$$
Z(f)=\left\{x \in(0,1) ; \exists \varepsilon>0 \forall n \geq 0 ; f^{n} \mid(x-\varepsilon, x+\varepsilon) \text { is strictly monotone }\right\} .
$$

Clearly, $A(K, f)$ and $Z(f)$ are open and $A(R, f)$ is a $G_{\delta}$ set. Moreover if $K$ is a transitive $f$-cycle and $R$ is a register shift, then $A(K, f) \cap A(R, f)=\emptyset$ and $A(K, f) \cap Z(f)=\emptyset$. In general it can happen that $R \cap Z(f) \neq \emptyset$ and so $A(R, f) \cap Z(f) \neq \emptyset$. (For more details about the facts mentioned above see [1] or $[2]$.)

Now we can formulate Theorem A on the asymptotic behavior of a point under a piecewise monotone map.

Theorem A. ([1], [2]). Let $f \in M_{n}$ and $K_{1}, \ldots, K_{r}$ be transitive $f$-cycles and $R_{1}, \ldots, R_{s}$ be register shifts. Then the set

$$
\Lambda(f)=A\left(K_{1}, f\right) \cup \cdots \cup A\left(K_{r}, f\right) \cup A\left(R_{1}, f\right) \cup \cdots \cup A\left(R_{s}, f\right) \cup Z(f)
$$

is of type $G_{\delta}$ dense in $I$.

The following results give more information about the behavior of a "typical" orbit of $x \in I$ with respect to $f \in \tilde{M}_{n, 1}$. 
Theorem B.. Let $f \in \tilde{M}_{n, c}$ for $c>1$. Then $f$ has no register shift and $Z(f)=\emptyset$.

If we consider the space $\left(\tilde{M}_{n, 1}, \varrho\right)$ we have the following contrary results.

Theorem C.. A typical function from $\tilde{M}_{n, 1}$ has no transitive $f$-cycle and $Z(f)=\emptyset$.

Theorem D.. A typical function from $M_{n, 1}$ has no transitive $f$-cycle and $Z(f)=\emptyset$.

\section{$3 \quad$ Residual set in $\left(\tilde{M}_{n, 1}, \varrho\right)$}

We start this section with some auxiliary results. We will not prove all of these facts. Let $n \geq 1$ be fixed and $\tilde{M}_{n, 0}$ be the closure of $M_{n}$ in the space $(C(I), \varrho)$.

Proposition 3.1.. $\left(\tilde{M}_{n, 0}, \varrho\right)$ is a complete metric space.

Proposition 3.2.. For $c \geq 0$ the set $\tilde{M}_{n, c}$ is closed in $\left(\tilde{M}_{n, 0}, \varrho\right)$.

Proposition 3.3.. For $c \geq 0$ the set $M_{n, c}$ is of type $G_{\delta}$ dense in $\left(\tilde{M}_{n, c}, \varrho\right)$.

Proof. Let $[a, b]$ be an interval in $I$. Obviously the sets

$$
K_{ \pm}(a, b)=\left\{f \in \tilde{M}_{n, c} ; f^{\prime}(x)= \pm c \quad \text { for } x \in(a, b)\right\}
$$

are closed and nowhere dense. Let $\left\{\left[a_{k}, b_{k}\right]\right\}_{k=0}^{\infty}$ be a sequence of intervals such that for any interval $J \subset I$ there is a $k \geq 0$ such that $\left[a_{k}, b_{k}\right] \subset J$. Then $M_{n, c}=\tilde{M}_{n, c} \backslash \bigcup_{k=0}^{\infty}\left(K_{+}\left(a_{k}, b_{k}\right) \cup K_{-}\left(a_{k}, b_{k}\right)\right)$ and proof is finished.

The following assertion is an easy consequence of Propositions 3.1., 3.2., and 3.3.

Lemma 3.4.. If $f \in M_{n, 1}$, then $Z(f)=\emptyset$ and for a typical function from $\tilde{M}_{n, 1}$ we have $Z(f)=\emptyset$.

The following corollary is immediate.

Corollary 3.5.. If $f \in \tilde{M}_{n, 1}$ and $R$ is a register shift, then $\operatorname{int}(R)=\emptyset$.

Proof. It suffices to observe that $\operatorname{int}(R) \backslash Z(f)$ is countable for any $f \in$ $M_{n}$. 
There exist functions in $M_{n, c}$ whose turning points are either periodic or eventually periodic points. Hence let

$$
\begin{aligned}
& P_{n, c}=\left\{f \in M_{n, c} ; T(f)=A \cup B,\right. \\
& \left.\quad(A \subset \operatorname{Per}(f)) \&\left(\forall x \in B \exists k \in \mathbb{N} ; f^{k}(x) \in A\right)\right\} .
\end{aligned}
$$

Lemma 3.6.. If $c \geq 1$, then the set $P_{n, c}$ is dense in $\left(\tilde{M}_{n, c}, \varrho\right)$.

Proof. Choose an open set $U$ in $\tilde{M}_{n, c}$. By Proposition 3.3. there exists function $f \in U \cap M_{n, c}$ and if we denote the turning points of $f$ by $z_{1}<z_{2}<$ $\cdots<z_{m}(m \leq n)$, then without loss of generality we can assume that

$$
f\left(z_{i}\right) \notin\{0,1\} \quad \text { for } \quad i \in\{1, \ldots, m\} .
$$

Since $c \geq 1$, the set $\bigcup_{n=0}^{\infty} f^{-n}(T(f))$ is dense in $I$. Suppose $f$ has at $z_{1}$ a local maximum (The opposite case is analogous.) The reader can easily verify that there exists $g_{1} \in U \cap M_{n, c}$ such that $T\left(g_{1}\right)=T(f), g_{1}\left(z_{j}\right)=f\left(z_{j}\right)$ for $j \neq 1$ $g_{1}\left(z_{1}\right) \geq f\left(z_{1}\right)$ (see $\left.(*)\right)$ and $g_{1}\left(z_{1}\right) \in \bigcup_{n=0}^{\infty} g_{1}^{-n}\left(T\left(g_{1}\right)\right)$. Obviously we have $g_{1}^{k}\left(z_{1}\right)=z_{i}$ for some $k \geq 1$ and $i \in\{1, \ldots, m\}$. Moreover $g_{1}$ can be chosen such that condition $(*)$ also holds for $g_{1}$. So we can repeat this procedure for $z_{2}, \ldots, z_{m}$ and finally we get a function $g \in U \cap M_{n, c}$ such that $T(g)=T(f)$ and for any $z \in T(g)$ there is a $k \geq 1$ such that $g^{k}(z) \in T(g)$. Hence obviously $g \in P_{n, c}$. (See figure 1.)

Remark 3.1. If $c \in[0,1)$, then the set $P_{n, c}$ is not dense in $\left(\tilde{M}_{n, c}, \varrho\right)$.

For what follows let $R_{m}$ be a finite set of disjoint closed intervals such that the sum of their lengths is less than $1 / \mathrm{m}$. Analogous to the definition of $P_{n, c}$ for $f \in \tilde{M}_{n, 1}$ let $f \triangle R_{m}$ denote the statement that there is a partition of $T(f)$ into two disjoint parts $A_{f}, B_{f}$ such that

(i) for all $x \in A_{f}$ there is $J \in R_{m}$ such that $x \in \operatorname{int}(J)$,

(ii) for all $J \in R_{m}$ there is $k \in \mathbb{N}$ such that $f^{k}(J) \subset \operatorname{int}(J)$,

(iii) for all $x \in B_{f}$ there is $J \in R_{m}$ and $k \in \mathbb{N}$ such that $f^{k}(x) \in \operatorname{int}(J)$.

Let $H_{m}=\left\{f \in \tilde{M}_{n, 1} ; f \triangle R_{m}\right.$ for some $\left.R_{m}\right\}$.

Lemma 3.7.. The set $H=\bigcap_{m=1}^{\infty} H_{m}$ is of type $G_{\delta}$ dense in $\left(\tilde{M}_{n, 1}, \varrho\right)$. 


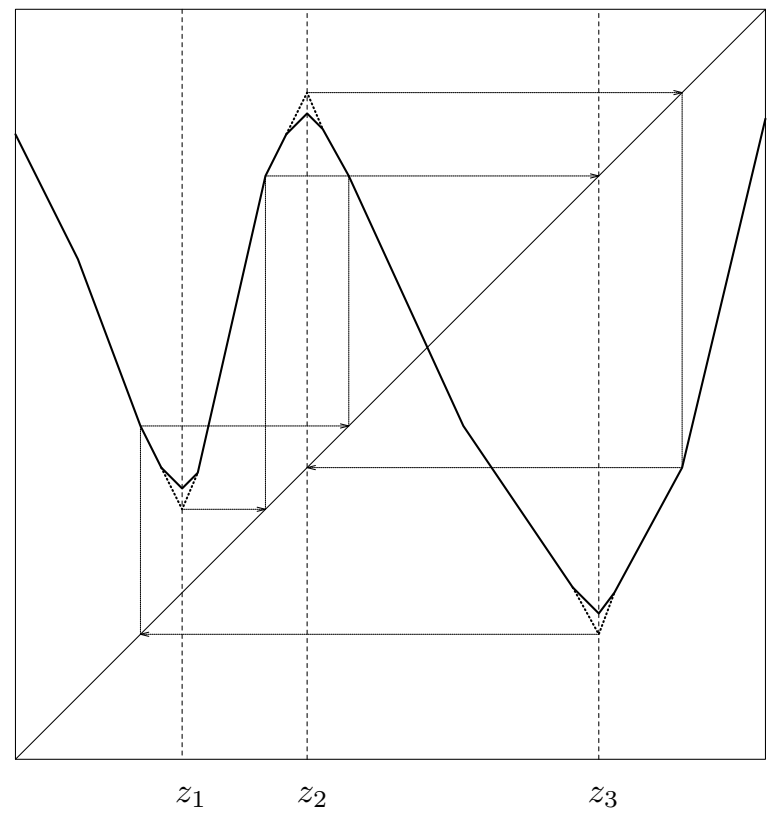

Figure 1: Functions $f$ and $g$ (dotted).

Proof. By Propositions 3.1. and 3.2. it suffices to show that the set $H_{m}$ is open and dense in $\left(\tilde{M}_{n, 1}, \varrho\right)$. The first property is clear from (i)-(iii) and in order to prove the second one we will use Lemma 1.6.

Choose an open set $U$ in $\tilde{M}_{n, 1}$. By Lemma 3.6. there is a function $f \in$ $U \cap P_{n, 1}$ such that $f(T(f)) \subset(0,1)$. Let $A_{f}=T(f) \cap \operatorname{Per}(f), B_{f}=T(f) \backslash A_{f}$, let $C_{f}=\left\{f^{k}\left(A_{f}\right)\right\}_{k=0}^{\infty}$ and let $D_{f}=\left\{f^{k}\left(B_{f}\right)\right\}_{k=0}^{\infty}$. Set $C_{f} \cup D_{f}=\left\{x_{1}, \ldots, x_{q}\right\}$ where $q=\operatorname{card}\left(C_{f} \cup D_{f}\right)$. Then there is a union of disjoint intervals $V=$ $\bigcup_{i=1}^{q}\left(c_{i}, d_{i}\right)$ such that $x_{i} \in\left(c_{i}, d_{i}\right)$ and $\sum_{i=1}^{q}\left(d_{i}-c_{i}\right)<1 / m$. For $0<\alpha<$ $\min _{i=1}^{q}\left\{\left|x_{i}-c_{i}\right|,\left|x_{i}-d_{i}\right|\right\}$ we also have $V_{\alpha} \subset V$ where $V_{\alpha}=\bigcup_{i=1}^{q}\left[x_{i}-\alpha, x_{i}+\alpha\right]$. Let $g \in C(I)$ be such that

(iv) $g(x)=f(x)$ for $\quad x \in C_{f} \cup D_{f}$,

(v) $T(g)=T(f)$,

(vi) $\left|g^{\prime}(x)\right|=1$ for $x \in V_{\alpha}$,

(vii) $g(x)=f(x)$ for $\quad x \in I \backslash V$,

(viii) $g \mid J$ is linear for any interval $J \subset V \backslash V_{\alpha}$. 
It is easy to verify that $g$ is unique and $g \in \tilde{M}_{n, 1}$. We can choose $V$ and $\alpha$ small enough such that $g \in U$. In addition we have $X_{g}=X_{f}$ for $X \in\{A, B, C, D\}$. Let $R_{m}=\left\{[x-\alpha, x+\alpha] ; x \in A_{g}\right\}$. From (vi) we have $g([x-\alpha, x+\alpha]) \subset$ $[g(x)-\alpha, g(x)+\alpha]$ for any $x \in C_{g} \cup D_{g}$ and so from (iv)-(vi) for the partition $T(g)=A_{g} \cup B_{g}$ we have that $g \triangle R_{m}$ is nearly fulfilled. More precisely it is fulfilled except for (ii) where we have only $f^{k}(J) \subset J$ instead of $f^{k}(J) \subset \operatorname{int}(J)$.

Observe that $C_{g}$ is a finite union of orbits of some turning points, so we can write $C_{g}=\bigcup_{i=1}^{s} \operatorname{orb}\left(x_{i}\right)$ where $x_{i} \in T(g)$ and $\operatorname{orb}\left(x_{i}\right) \cap \operatorname{orb}\left(x_{j}\right)=\emptyset$ for any $1 \leq i \neq j \leq s$. Now we will modify $g$ in a neighborhood of $x_{i}$ in order to get new function a $h$ and a set $R_{m}$ such that $h \triangle R_{m}$.

Let $k_{i}=\operatorname{per}\left(x_{i}\right)$ for $1 \leq i \leq s$. Because $x_{i} \in T(g)$ it is easy to see from (vi) that either

$$
g^{k_{i}}\left(\left[x_{i}-\alpha, x_{i}+\alpha\right]\right)=\left[x_{i}-\alpha, x_{i}\right]
$$

or

$$
g^{k_{i}}\left(\left[x_{i}-\alpha, x_{i}+\alpha\right]\right)=\left[x_{i}, x_{i}+\alpha\right] .
$$

Suppose (1). Then obviously for any $x \in \operatorname{orb}\left(x_{i}\right) \cap T(g)$ if $g^{k}([x-\alpha, x+\alpha]) \subset$ $\left[x_{i}-\alpha, x_{i}+\alpha\right]$ for some $k \geq 1$, then $g^{k}([x-\alpha, x+\alpha]) \subset\left[x_{i}-\alpha, x_{i}\right]$. Hence for any $J \in R_{m}$ there is $k \geq 0$ (we will take the minimal one) and $1 \leq i \leq s$ such that

$$
g^{k}(J) \subset\left[x_{i}-\alpha, x_{i}\right]
$$

Similarly for (2).

Because $f \in M_{n, 1}$ we have that

$$
\left|\frac{g\left(c_{i}\right)-g\left(x_{i}-\alpha\right)}{c_{i}-\left(x_{i}-\alpha\right)}\right|>1 \quad \text { and } \quad\left|\frac{g\left(d_{i}\right)-g\left(x_{i}+\alpha\right)}{d_{i}-\left(x_{i}+\alpha\right)}\right|>1 \text {. }
$$

So there is $0<\lambda<\min \left\{\alpha,\left(x_{i}-\alpha\right)-c_{i}, d_{i}-\left(x_{i}+\alpha\right)\right\}$ such that for any $1 \leq i \leq s$

$$
\left|\frac{g\left(c_{i}\right)-g\left(x_{i}-\alpha\right)}{\left(x_{i}-\alpha+\lambda\right)-c_{i}}\right|>1 \quad \text { and } \quad\left|\frac{g\left(d_{i}\right)-g\left(x_{i}+\alpha\right)}{d_{i}-\left(x_{i}+\alpha-\lambda\right)}\right|>1 .
$$

Now we can define function $h$ and a new set $R_{m}$. Let

(ix) $h(x)=g(x)$ for any $x \in I \backslash \bigcup_{i=1}^{s}\left(c_{i}, d_{i}\right)$,

(x) if (1), then $h(x)=g(x+\lambda)$ for any $x \in\left[x_{i}-\alpha-\lambda, x_{i}+\alpha-\lambda\right]$, if $(2)$, then $h(x)=g(x-\lambda)$ for any $x \in\left[x_{i}-\alpha+\lambda, x_{i}+\alpha+\lambda\right]$,

(xi) if (1), then $h$ is linear on $\left[c_{i}, x_{i}-\alpha-\lambda\right]$ and $\left[x_{i}+\alpha-\lambda, d_{i}\right]$, if (2), then $h$ is linear on $\left[c_{i}, x_{i}-\alpha+\lambda\right]$ and $\left[x_{i}+\alpha+\lambda, d_{i}\right]$, 


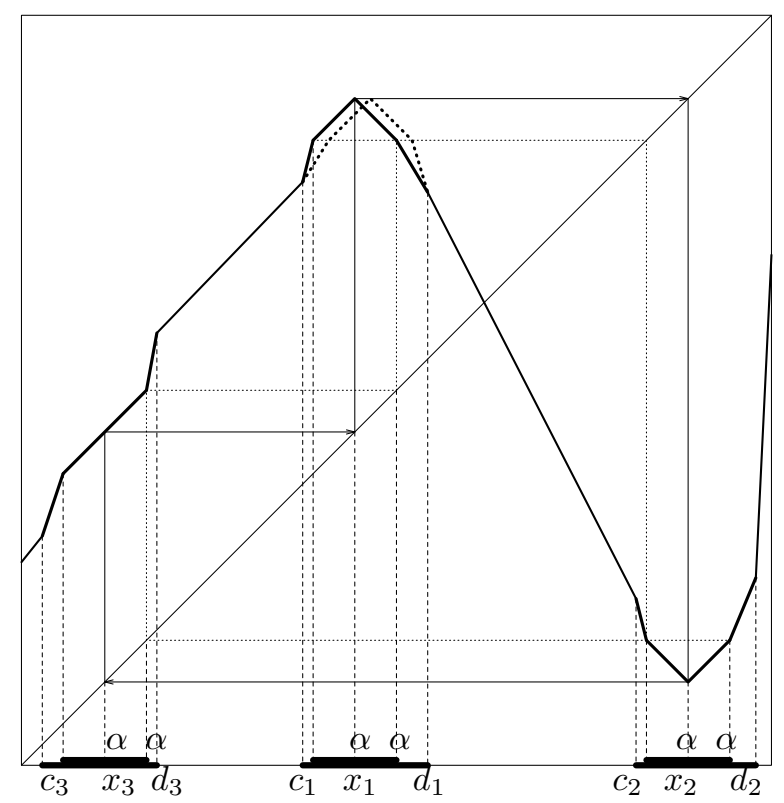

Figure 2: Functions $g$ and $h$ (dotted).

and in $R_{m}$ we replace interval $\left[x_{i}-\alpha, x_{i}+\alpha\right]$ by $\left[x_{i}-\alpha-\lambda, x_{i}+\alpha-\lambda\right]$ in case (1) and by $\left[x_{i}-\alpha+\lambda, x_{i}+\alpha+\lambda\right]$ in case (2). (See figure 2.)

From (4) it follows that $h \in \tilde{M}_{n, 1}$ and we can choose $\lambda>0$ small enough such that $h \in U$.

For $1 \leq i \leq s$ let $z_{i}=x_{i}-\lambda$ and $J_{i}=\left[x_{i}-\alpha-\lambda, x_{i}+\alpha-\lambda\right]$ in case (1) or $z_{i}=x_{i}+\lambda$ and $J_{i}=\left[x_{i}-\alpha+\lambda, x_{i}+\alpha+\lambda\right]$ in case (2).

Let $B_{h}=B_{g}$ and $A_{h}=\left(A_{g} \backslash \bigcup_{i=1}^{s} x_{i}\right) \cup\left\{z_{i}\right\}_{i=1}^{s}$. We have $T(h)=A_{h} \cup B_{h}$ and $A_{h} \cap B_{h}=\emptyset$. Moreover, for any $x \in B_{h}$ there is $k \geq 1$ (we will take the minimal one) such that $g^{k}(x)=x_{i}$ for some $1 \leq i \leq s$. But then $h^{k}(x)=x_{i}$ and $x_{i} \in \operatorname{int} J$ for some $J \in R_{m}$. So condition (iii) is fulfilled and condition (i) is obvious. Only (ii) remains.

From (3) we have that for any $J \in R_{m}$ there is $k \geq 0$ such that $h^{k}(J) \subset J_{i}$ for some $1 \leq i \leq s$. But $h(J)=g(J)$ for any $J \neq J_{i}$ and $h\left(J_{i}\right)=g\left(\left[x_{i}-\alpha, x_{i}+\right.\right.$ $\alpha]$ ). Hence if $J=J_{i}$, then $h^{k_{i}}\left(J_{i}\right) \subset \operatorname{int}\left(J_{i}\right)$ by (1), (2). If $J \neq J_{i}$ and $x \in J \cap A_{h}$, then $h^{k_{i}}(J) \subset[x-\alpha+\lambda, x+\alpha-\lambda] \subset \operatorname{int}(J)$ by (vi), (x). So we have $h \triangle R_{m}$. 


\section{Proofs of Theorems}

Theorem B.. Let $f \in \tilde{M}_{n, c}$ for $c>1$. Then $f$ has no register shift and $Z(f)=\emptyset$.

Proof. (Compare with [3].) By Lemma 1.4 and Corollary 1.5 it suffices to show that there is $\eta>0$ such that the length of $f^{k}(J)$ is greater than $\eta$ for any interval $J \subset I$ and a suitable $k \in \mathbb{N}(k=k(J))$. Let $c^{m}>2$. Then any interval mapped by $f^{m}$ will expand while it does not contain at least two points of $T\left(f^{m}\right)$.

Theorem C.. A typical function from $\tilde{M}_{n, 1}$ has no transitive $f$-cycle and $Z(f)=\emptyset$.

Proof. Consider $f \in H$ (see Lemma 3.7.). Assume that $f$ has a transitive $f$-cycle $K$. Then $K \cap T(f) \neq \emptyset$. And by (i)-(iii) there exists a closed nondegenerate interval $J \subset K$ such that $f^{k}(J) \subset J$ for some $k \in \mathbb{N}$ and $\operatorname{orb}(J) \neq K$. But this contradicts our assumption that $K$ is transitive $f$-cycle. Second part follows from Lemma 3.4..

Theorem D.. A typical function from $M_{n, 1}$ has no transitive $f$-cycle and $Z(f)=\emptyset$.

Proof. The assertion easily follows from Propositions 3.1.-3.3. and Theorem C.

\section{Construction of a Function From $M_{n, 1}$ That Has No Transitive $f$-cycle}

Let $A=\left\{a_{j}\right\}_{j=1}^{\infty}$ and $p_{i}=\prod_{j=1}^{i} a_{j}$. We say that function $f$ has an $A$-register shift if there is a sequence $\left\{J_{i}\right\}_{i=1}^{\infty}$ of subintervals of $I$ such that $J_{i}$ is periodic with $\operatorname{per}\left(J_{i}\right)=p_{i}$ and $J_{i+1} \subset J_{i}$ for all $i \in \mathbb{N}$.

We denote by $|S|$ the Lebesgue measure of a set $S$, by $\operatorname{conv}(S)$ the convex hull of $S$ and by d $\left(S_{1}, S_{2}\right)$ the distance between the sets $S_{1}, S_{2}$. Moreover, we say $S_{1}<S_{2}$ if $x<y$ for any $x \in S_{1}, y \in S_{2}$.

Lemma 5.1.. For any $A=\left\{a_{i}\right\}_{i=1}^{\infty}$ there is $f \in M_{1,1}$ such that $f$ has $A$-register shift.

Proof. Fix an $A=\left\{a_{i}\right\}_{i=1}^{\infty}$. Without loss of generality we can assume that $a_{i}$ is a prime number for all $i \in \mathbb{N}$. 
Let $a \in \mathbb{N}$ be prime. If $a>2$, then define $\psi:\{1, \ldots, a\} \rightarrow\{1, \ldots, a\}$ by

$$
\begin{aligned}
& \psi(1)=\frac{1}{2}(a+1), \\
& \psi(i)=a+2-i \text { for } 1<i \leq \frac{1}{2}(a+1), \\
& \psi(i)=a+1-i \text { for } \frac{1}{2}(a+1)<i \leq a,
\end{aligned}
$$

and if $a=2$, then simply $\psi(1)=2$ and $\psi(2)=1$.

Let $\left\{I_{i}^{a}\right\}_{i=1}^{a}$ be the set of subintervals of $I=[0,1]$ such that

$$
\begin{array}{rlrl}
I & =\operatorname{conv}\left(\bigcup_{i=1}^{a} I_{i}^{a}\right), & & \\
\left|I_{i}^{a}\right| & =\left|I_{j}^{a}\right| & & \text { for } 1 \leq i, j \leq a, \\
I_{i}^{a} & <I_{i+1}^{a} & & \text { for } 1 \leq i<a, \\
\mathrm{~d}\left(I_{i}^{a}, I_{i+1}^{a}\right) & <\mathrm{d}\left(I_{\psi(i)}^{a}, I_{\psi(i+1)}^{a}\right) & \text { for } 1 \leq i<a .
\end{array}
$$

Of course this is possible only if $a>2$. If $a=2$ let $I_{2}^{2}=\left[0, \frac{1}{3}\right], I_{1}^{2}=\left[\frac{2}{3}, 1\right]$. This change in order of indexing saves some troubles when $a=2$.

We can assume that $\sum_{i=1}^{a}\left|I_{i}^{a}\right| \leq \frac{2}{3}$. Let $g_{a}$ be a continuous function such that

- $g_{a}\left(I_{i}^{a}\right)=I_{\psi(i)}^{a}$ for $1 \leq i \leq a$,

- $g_{a} \mid I_{i}^{a}$ is linear for $i \neq 2$,

- $g_{a} \mid J$ is linear for any interval $J \subset I$ with $J \cap I_{i}^{a}=\emptyset$ for $i=1, \ldots, a$,

- we do not specify $g_{a} \mid I_{2}^{a}$,

- $g_{a}$ can have only one turning point.

(See figure 3.)

Let $f \in C(I)$ and $f^{*}(x)=f(1-x)$ for $x \in I$. (The graph of $f^{*}$ is symmetric to the graph of $f$ in the axis $x=\frac{1}{2}$.)

Now for $i \geq 1$ let $f_{i}$ be a function such that $f_{i}=g_{a_{i}}$ and moreover $f_{i} \mid I_{2}^{a_{i}}$ "looks like" function $f_{i+1}^{*}$ (this mean that $h_{1} \circ f_{i} \mid I_{2}^{a_{i}} \circ h_{2}=f_{i+1}^{*}$ where $h_{1}$ is linear increasing mapping from $I_{a_{i}}^{a_{i}}$ onto $I$ and $h_{2}$ is linear increasing mapping from $I$ onto $I_{2}^{a_{i}}$ ).

Henceforth, if we say "slope" we mean in fact "absolute value of the slope".

Claim. Function $f_{i}$ has the following properties: 

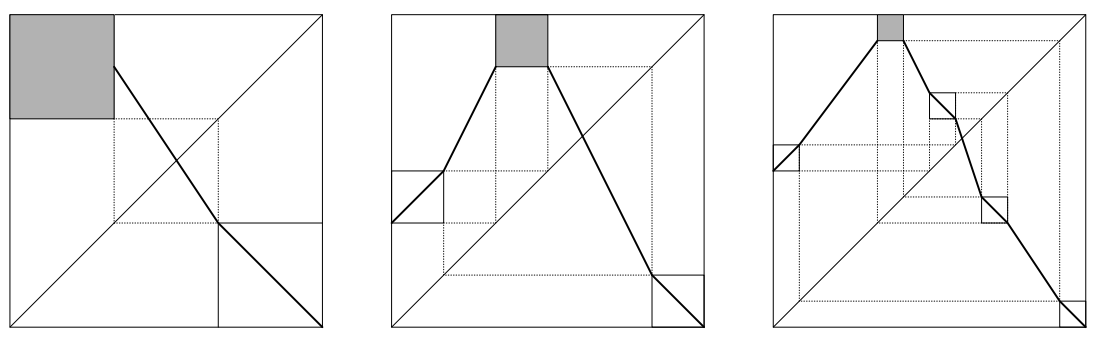

Figure 3: Possible functions $g_{2}, g_{3}$ and $g_{5}$.

(1) $f_{i} \mid J$ is linear with slope greater than 1 for any interval $J \subset I$ such that $J \cap I_{i}^{a_{i}}=\emptyset$ for all $i=1, \ldots, a_{i}$,

(2) If $A_{i}=\left\{a_{j}\right\}_{j=i}^{\infty}$, then $f_{i}$ has $A_{i}$-register shift,

(3) $A_{1}$-register shift of $f_{1}$ is generated by $\left\{J_{i}\right\}_{i=1}^{\infty}$ where $\left|\operatorname{orb}\left(J_{i}\right)\right| \leq\left(\frac{2}{3}\right)^{i}$,

(4) $f_{i}$ has a unique turning point.

Proof. Part (1) is obvious because $g_{a}(0)>0$ and so there is no difficulty even if $a_{i}=2$. Interval $I_{j}^{a_{i}}$ is periodic with period $a_{i}$ and $f_{i}^{a_{i}} \mid I_{j}^{a_{i}}$ is exactly function $f_{i+1}$ because $I_{j}^{a_{i}}$ is mapped once by $f_{i} \mid I_{2}^{a_{i}}$ which is $f_{i+1}^{*}$, once by the order preserving linear homeomorphism $f_{i} \mid I_{1}^{a_{i}}$ (or zero times if $a_{i}=2$ ) and $a_{i}-2$ times by the order reversing linear homeomorphism $f_{i} \mid I_{j}^{a_{i}}$ for $2<j \leq a_{i}$ (or once by $f_{i} \mid I_{1}^{a_{i}}$ if $a_{i}=2$ ). Hence we have part (2). Parts (3) and (4) are obvious.

Therefore $f_{1} \in \tilde{M}_{1,1}$ and it has an $A$-register shift. Let $J_{0}=I, J_{1}=I_{2}^{a_{1}}$ and $J_{i}$ be the interval corresponding to $I_{2}^{a_{i}}$ if we consider only $f_{1} \mid J_{i-1}$. More precisely $J_{i}$ is a periodic interval with period $p_{i}$ such that $J_{i} \cap T\left(f_{1}\right) \neq \emptyset$.

Here is our strategy for obtaining a function $f \in M_{1,1}$ such that $f$ has an $A$-register shift.

1. Let $F_{1}=f_{1}$. Then $\left\{J_{i}\right\}_{i=1}^{\infty}$ is our sequence of periodic intervals which generate $A$-register shift. Moreover $F_{1} \mid J$ is linear with slope greater than 1 for any interval such that $J \cap \operatorname{orb}\left(J_{1}\right)=\emptyset$ (see Claim).

2. Assume that $F_{n} \mid J$ is linear with slope greater than 1 for any interval $J$ such that $J \cap \operatorname{orb}\left(J_{n}\right)=\emptyset$. We will modify $F_{n}$ on the set $\operatorname{orb}\left(J_{n-1}\right)$ such that we will obtain new intervals $J_{i}$ for $i \geq n$ and our modified function $F_{n+1}$ will be linear with slope greater than 1 on any interval $J$ such that $J \cap \operatorname{orb}\left(J_{n+1}\right)=\emptyset$. 

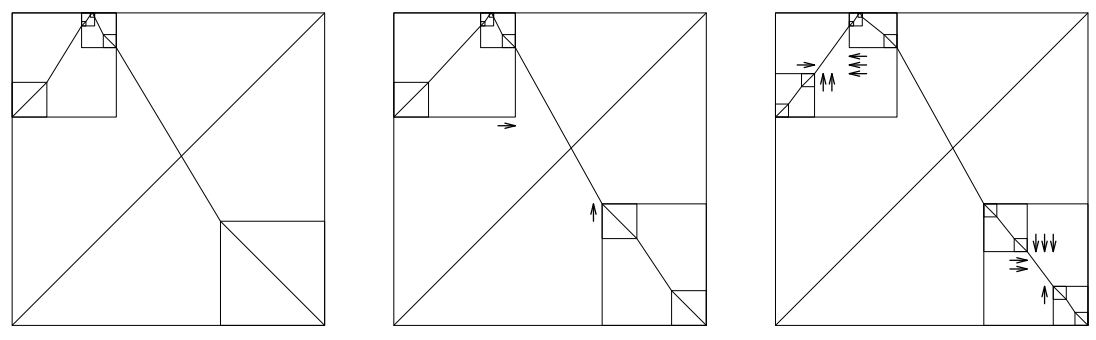

Figure 4: Illustration how to get $F_{1}, F_{2}, F_{3}$ for $A=\{2,2,2, \ldots\}$.

3. Finally we will get function $f=\lim _{n \rightarrow \infty} F_{n}$.

Fix $n \in \mathbb{N}$. Let $\operatorname{orb}\left(J_{n}\right)=\left\{I_{i}^{n}\right\}_{i=1}^{p_{n}}$ where $I_{1}^{n}=J_{n}$ and $F_{n}\left(I_{i}^{n}\right)=I_{i+1}^{n}$ $\left(F_{n}\left(I_{p_{n}}^{n}\right)=I_{1}^{n}\right)$. Let $\operatorname{orb}\left(J_{n+1}\right)$ equal the set of intervals $\left\{I_{i, j}=\left[a_{i, j}, b_{i, j}\right]\right\}$ where $1 \leq i \leq p_{n}, 1 \leq j \leq a_{n+1}, I_{i, j} \subset I_{i}^{n}$ and $b_{i, j}<a_{i, j+1}$. Of course $I_{i}^{n}=\left[a_{i, 1}, b_{i, a_{n+1}}\right]$.

We have that $\left|I_{i}^{n}\right|=\left|I_{j}^{n}\right|$ for any $i, j$ and $F_{n} \mid I_{i}^{n}$ is either linear with slope \pm 1 (if $i>1$ ) or "looks like" function $f_{n+1}$ or $f_{n+1}^{*}$ (if $i=1$ ). (All this is true for $n=1$ and our modification will preserve these properties.)

Let $F_{n+1}\left|S=F_{n}\right| S$ for $S=I \backslash \operatorname{orb}\left(J_{n-1}\right)$ and we will define $F_{n+1}$ on $\operatorname{orb}\left(J_{n-1}\right)$. Take $c>1$ and new intervals $I_{i}^{*} \subset \operatorname{orb}\left(J_{n-1}\right)$ such that $I_{2}^{*}=I_{2}^{n}$, $I_{i}^{n} \subset I_{i}^{*}$ for $1 \leq i \leq p_{i},\left|I_{i+1}^{*}\right|=c\left|I_{i}^{*}\right|$ for $2 \leq i<p_{i}$ and $\left|I_{1}^{*}\right|=c\left|I_{p_{i}}^{*}\right|$. We can choose $c$ so small that the intervals $I_{i}^{*}$ are pairwise disjoint. Let $F_{n+1}\left(I_{i}^{*}\right)=I_{i+1}^{*}\left(F_{n+1}\left(I_{p_{i}}^{*}\right)=I_{1}^{*}\right)$ and let $F_{n+1}$ be linear outside the intervals $I_{i}^{*}$. Choose $a_{i, j}^{*}, b_{i, j}^{*} \in I_{i}^{*}$ such that

$$
\begin{aligned}
& I_{i}^{*}=\left[a_{i, 1}^{*}, b_{i, a_{n+1}}^{*}\right], \\
& a_{i, j}^{*}<b_{i, j}^{*}<a_{i, j+1}^{*}<b_{i, j+1}^{*} \text { for } 1 \leq j<a_{n+1}, \\
& b_{i, j}^{*}-a_{i, j}^{*}=b_{i, j}-a_{i, j} \quad \text { for } 1 \leq j \leq a_{n+1}, \\
& a_{i, j+1}^{*}-b_{i, j}^{*}=k\left(a_{i, j+1}-b_{i, j}\right) \text { for } 1 \leq j<a_{n+1}
\end{aligned}
$$

for some constant $k>1$ and let $I_{i, j}^{*}=\left[a_{i, j}^{*}, b_{i, j}^{*}\right]$. Now we can complete the definition of $F_{n+1}$.

If $F_{n}\left(I_{i, j}\right)=I_{i+1, s}$, then $F_{n+1}\left(I_{i, j}^{*}\right)=I_{i+1, s}^{*}$ and the graph of $F_{n+1}$ on $I_{i, j}^{*}$ will be the same as the graph of $F_{n}$ on $I_{i, j}$. (It will be linear with slope 1 unless $I_{i, j}=J_{n+1}$ when it will "look like" $f_{n+2}$ or $f_{n+2}^{*}$.) And let $F_{n+1}$ be linear outside the intervals $I_{i, j}^{*}$. (See figure 4.) 
So $F_{n+1}$ is completely defined. Let the new $J_{n}=I_{1}^{*}, J_{n+1}=I_{1, s}^{*}$ (where

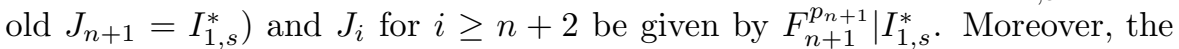
slopes of $F_{n+1}$ may be decreased comparing to the slopes of $F_{n}$ only on the set $\left(\operatorname{orb}\left(J_{n-1}\right) \backslash \operatorname{orb}\left(J_{n}\right)\right) \cup\left(J_{n} \backslash \operatorname{orb}\left(J_{n+1}\right)\right)$ where they were bigger than 1 and so it is possible to choose $c>1$ such that the changes are small enough and the slopes remain greater than 1 . And finally it is obvious that the slopes of $F_{n+1}$ on the set $\operatorname{orb}\left(J_{n}\right) \backslash\left(\operatorname{orb}\left(J_{n+1}\right) \cup J_{n}\right)$ are now greater than $c$. Hence we made the required modification.

Moreover we can choose $c>1$ sufficiently small such that $\left|\operatorname{orb}\left(J_{n}\right)\right|$ increases during this modification no more than twice. And obviously for $i>n$ $\left|\operatorname{orb}\left(J_{i}\right)\right|$ remains the same. So we made modification on an invariant set $S$ where $|S| \leq 2\left(\frac{2}{3}\right)^{n-1}$ and this set remains invariant. This proves that $\lim _{n \rightarrow \infty} F_{n}=f$ exists and is continuous. It is obvious that $f \in M_{1,1}$ and $f$ has an $A$-register shift.

Corollary 5.2.. The function $f$ from Lemma 5.1 has no transitive $f$-cycle.

Proof. Each register shift and transitive $f$-cycle are disjoint and they must contain a turning point. But our $f$ has only one turning point and a register shift.

Remark 5.1. For the construction of $f \in \tilde{M}_{n, c}$ for $c \in[0,1]$, see [1], [4].

\section{References}

[1] C. Preston, Iterates of piecewise monotone mappings on an interval, Lecture Notes in Mathematics 1347, Springer-Verlag, 1988.

[2] A. Blokh, Decompositions of dynamical systems on an interval, Russ. Math. Surveys 38 (1983), 133-134.

[3] K. Janková, M. Polakovič, Transitivity of expanding maps of the interval, (Russian and Slovak summaries). Acta Math. Univ. Comenian. 56/57 (1989), 243-247.

[4] Z. Nitecki, Topological dynamics on the interval, Ergodic theory and dynamical systems II, 1-73, Progress in Math. 21, Birkhäuser, Boston, 1982. 